\title{
Awareness in contextual cuing with extended and concurrent explicit tests
}

\author{
Andrea C. Smyth ANd David R. SHANKS \\ University College London, London, England
}

\begin{abstract}
The term contextual cuing refers to improved visual search performance with repeated exposure to a configuration of objects. Participants use predictive cues - derived from learned associations between target locations and the spatial arrangement of the surrounding distractors in a configuration - to efficiently guide search behavior. Researchers have claimed that contextual cuing can occur implicitly. The present experiments examined two explicit measures-generation and recognition. In Experiment 1, we found that contextual cuing information was consciously retrievable when the number of trials used in a generation test was increased, and the results also suggested that the shorter tests that were used previously were not statistically powerful enough to detect a true awareness effect. In Experiment 2, concurrent implicit and explicit (generation and recognition) tests were employed. At a group level, learning did not precede awareness. Although contextual cuing was evident in participants who were selected post hoc as having no explicit awareness, and for specific configurations that did not support awareness, we argue that awareness may nevertheless be a necessary concomitant of contextual cuing. These results demonstrate that contextual cuing knowledge is accessible to awareness.
\end{abstract}

There has been an accumulation of research over the past 20 or so years that appears to suggest that human learning and memory are organized into at least two distinct systems - one of which is explicit, or declarative, and one of which is implicit, or procedural. The former system allows conscious recall of facts or events, whereas the latter influences performance unconsciously (Squire, 1992). Central to this systems view of learning and memory is a very large body of evidence indicating that the implicit system can be isolated in appropriate preparations - that is, in tasks in which learning proceeds independently of the individual's awareness of the learned properties of the materials. Such "implicit learning" tasks - which have been studied for many decades (Thorndike, 1931) - therefore have an important reciprocal relationship to memory systems theories.

Despite the enormous number of studies attempting to demonstrate implicit learning, it is fair to say that these have been consistently dogged by controversy. A common cycle of research begins with a new and apparently compelling instance of implicit learning, only for this instance to be undermined and challenged by later research. Examples include Thorndike's (1931) own studies of verbal operant conditioning (Dulany, 1961), Reber's artificial grammar experiments (Dulany, Carlson, \& Dewey, 1984), learning in the Iowa gambling task (Maia \& McClelland, 2004), studies of human conditioning (Lovibond \& Shanks, 2002), and studies of reaction times (RTs) to sequentially structured stimuli (Perruchet \& Amorim, 1992).

Chun and colleagues reported results from studies using a spatial contextual cuing paradigm (Chun \& Jiang,
1998) - a visual search task in which participants are shown displays containing a set of 12 letter stimuli and are required to detect a target stimulus (a letter T) within the subset of distractor stimuli (11 letter Ls), and which represents a new addition to the body of evidence for a dual-systems model of learning and memory. In contextual cuing experiments, the location of the target in half of the displays appears repeatedly with the same arrangement of the distractors surrounding it. Participants indirectly express evidence of implicitly learning to use the context of distractors as a cue for the location of the target by making faster responses to displays with this association, in comparison with responses made to novel displays that do not contain covariance between the location of the target and the surrounding distractor stimuli. The results of these experiments suggest that the contextual cuing effect occurs implicitly and outside of awareness, because when given a direct test of explicit knowledge - such as having to predict or generate the location of a missing target during a generation test (Chun \& Jiang, 2003) or making a recognition judgment (Chun \& Jiang, 1998; Chun \& Phelps, 1999; Howard, Howard, Dennis, Yankovich, \& Vaidya, 2004; Manns \& Squire, 2001; Park, Quinlan, Thornton, \& Reder, 2004) - participants perform no better than they would have through random guessing. The task is appealing because it appears to involve very "low-level" perceptual search processes, which phenomenologically seem outside awareness and conscious control.

Although superficially these results of a dissociation between direct (generation, recognition) and indirect (RT) measures of learning seem to demonstrate that the

A. C. Smyth, a.smyth@ucl.ac.uk 
dual-systems perspective is accurate in its partitioning of memory according to awareness at learning, a number of methodological problems have been raised against previous dissociations of this sort (Shanks \& St. John, 1994). Often when these problems are rectified, the finding of implicit learning without explicit access is not replicated (Shanks \& Johnstone, 1999; Shanks \& Perruchet, 2002).

One such issue relates to the power and reliability of the awareness test. The modal implicit learning finding is of above-chance performance on an indirect test-such as contextual cuing - and of chance performance on an explicit test. Plainly, this means that the inference of implicit learning rests on a null result in the awareness test, and the interpretation of such a null result depends critically on that test's power and reliability. Yet these awareness tests are rarely set up to guarantee adequate power/reliability. In typical contextual cuing experiments, although the indirect task comprises many hundreds of search trials to measure contextual cuing, only a small number of trials (e.g., 24) are presented in the recognition or generation tests (Chun \& Jiang, 1998, 1999, 2003; Howard et al., 2004). An "awareness effect" would have to be very large to be reliably detected in such a small number of trials. We address the issue of power in Experiment 1 by examining performance when the number of trials administered during the direct task is greatly increased to evaluate previous null results from measures of explicit knowledge.

The present study was concerned with addressing methodological issues in the spatial contextual cuing paradigm. In Experiment 1, we replicated the standard experimental procedure from Chun and Jiang (2003), but used a more powerful test of awareness. In Experiment 2, we presented repeated tests of awareness during the visual search task.

\section{EXPERIMENT 1}

In a generation test, participants see on each trial a display comprising 11 distractors arranged in a familiar (i.e., repeated) configuration. Instead of a target, however, a 12th distractor is presented at the location where the target would have appeared. Participants are asked to indicate which quadrant of the display they believe contains this substitute distractor - that is, where they believe the target would normally appear. Chun and Jiang (2003) argued that doing so constituted a test of awareness for the target location within that configuration, and that if performance on such a test was at chance while normal contextual cuing occurred, then that would demonstrate the implicitness of such cuing. Note that the response required in this test was quite different from that elicited in the cuing task itself (indicating whether the target is pointing left or right).

In the present experiment, we extended the generation task used in Chun and Jiang (2003) from 24 trials to 96 in order to examine whether the unconscious contextual knowledge learned during experiments in spatial contextual cuing is only accessible to implicit processes. If participants in past experiments did actually have explicit access to contextual knowledge from the detection task, we would expect to see the overall ability to generate target locations to emerge with the introduction of more trials in the generation task. Such a result would lead us to conclude that previous null explicit results can be attributed to inadequate probing for explicit knowledge in those experiments. Alternatively, if participants still show low generation performance after an extended test, then such a result would strengthen Chun and colleagues' claim that contextual cuing employs truly implicit processes.

\section{Method}

Participants. Forty-one University College London (UCL) undergraduates (22 women and 19 men) were recruited from the UCL psychology subject pool and were paid $£ 5$ for participating. None had taken part in any other contextual cuing experiments.

Design. The detection task was a $2 \times 24$ (configuration type $\times$ block) within-subjects design. Participants' RTs for detecting the target and accuracy in identifying the orientation of the target in the configuration were measured in each trial. The generation task was a $2 \times 4$ (configuration type $\times$ block) within-subjects design. Participants' ability to correctly generate the location of the target was measured for each configuration condition in each block.

Materials and Apparatus. The experiment was conducted using Visual Basic software that was designed to generate and present all stimuli, measure RTs, and record accuracy. The detection task contained 24 blocks of 24 trials each. On each trial, the participant viewed a configuration of letters and was asked to identify the orientation of the letter $\mathrm{T}$ in the display as quickly as possible. Half of the trials in each block displayed the same 12 configurations (repeated configurations) throughout the task, whereas the remaining trials contained configurations shown only once during the task (nonrepeated configurations). The presentation sequence of the configurations was randomized for each block. A unique set of 12 repeated configurations and 288 nonrepeated configurations was created for each participant using the criteria established in Chun and Jiang (1998) for the detection task. Each configuration contained $11 \mathrm{Ls}$ and $1 \mathrm{~T}$ shown against a gray background. All letters were presented in 30-point Arial font at a visual angle of $0.76^{\circ}$ at a viewing distance of approximately $60 \mathrm{~cm}$. The $21 \times 21 \mathrm{~cm}$ screen was divided into a four-quadrant matrix by dark gray dotted lines; a letter was randomly assigned to 1 of 16 possible spatial locations within a quadrant. Three letters colored red, green, yellow, or blue were randomly assigned to each quadrant, with each configuration containing three letters in each color. Each T was rotated $90^{\circ}$ to the right or left, and each $\mathrm{L}$ was shown at $0^{\circ}, 90^{\circ}$, $180^{\circ}$, or $270^{\circ}$. Figure 1 provides an example of the configurations shown during the task. The location and color of all letters in each repeated configuration was constant with each presentation, with the exception of the varying orientation of the letter T. The set of 12 nonrepeated configurations in each block of the detection task contained the same 12 spatial locations for the letter $\mathrm{T}$ in order to control for location probability effects. The 12 spatial locations for the letter $\mathrm{T}$ in both configuration conditions were also evenly distributed across the four quadrants of the screen.

The generation task followed the detection task and was composed of four blocks of 24 trials. The format was identical to the detection task: 12 repeated configurations and 12 nonrepeated configurations shown in a random sequence in each block. The repeated configurations were the same as those from the detection task, whereas a new set of 48 nonrepeated configurations was created for the generation task. The configurations in this phase of the experiment were only different from the detection task stimuli in that all Ts in the detection configurations were replaced with Ls.

Procedure. Each session began with instructions for the detection task: Locate the $\mathrm{T}$ in the configuration of letter Ls as quickly as possible; then respond to the direction of the T using the left and right arrows on the keyboard. Visual examples of the configurations and the two possible orientations of the target were included in the on-screen directions. Participants were not informed that some of the configurations would repeat across the experiment, nor were they given guidance to attend to the arrangement of letters. Famil- 

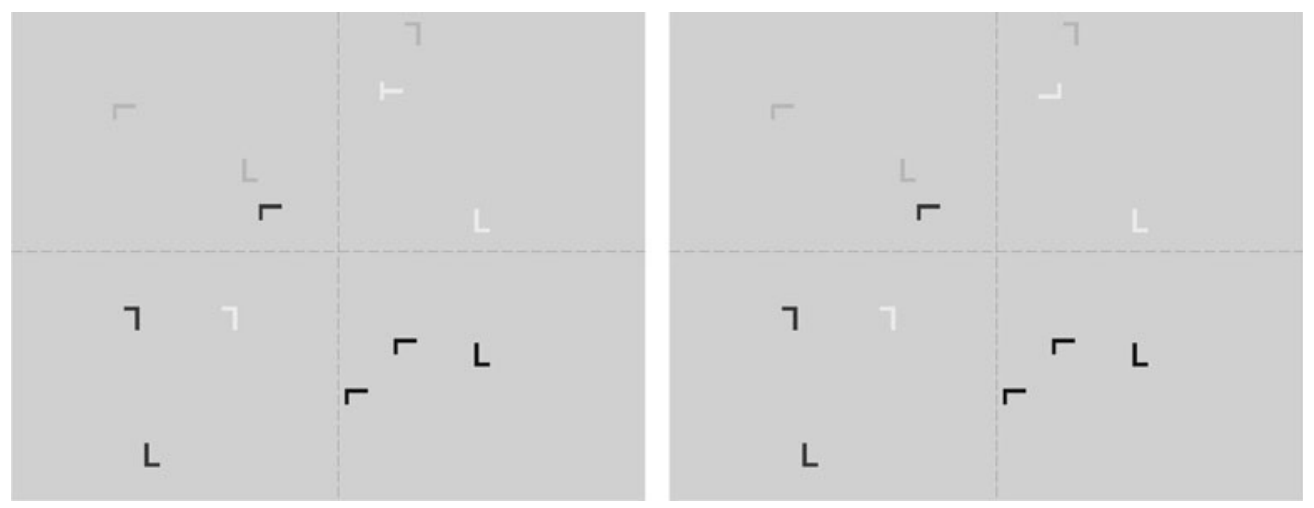

Figure 1. The configuration on the left is an example of a display shown during the detection task. The configuration on the right is the same display, but is altered to appear as it would during the generation task, in which participants must locate the region of the transformed $T$. The different levels of shading represent different colors (red, blue, green, yellow) in the actual display.

iarization with the task was established using six practice trials of configurations that were not shown during the main experiment.

Each configuration was preceded by an orienting white dot $(1 \times$ $1 \mathrm{~cm}$ ) for $1 \mathrm{sec}$ in the center of the screen. The program did not progress to the next trial until a valid response to the target orientation was given; then, the screen was cleared and auditory feedback was given according to the accuracy of the response. A high-pitched tone signified a correct answer, and a longer, low-pitched tone signified an incorrect answer. After each block of detection trials, participants received a break of at least $10 \mathrm{sec}$. Participants were allowed to progress to the next block by pressing the space bar, or they were free to continue resting if necessary after this interval.

After completing the 24 blocks in the detection task, awareness for the repeated configurations was assessed. Participants were asked, "During the experiment, do you think that any of the particular configurations of Ls were repeated?" Those who noticed a repetition received a follow-up question: "Approximately, when did you begin to notice this repetition?" Using a slider labeled by block from 1 to 24 , participants estimated the block in which awareness occurred before finally being asked, "After you realized particular configurations of Ls were being repeated, did you try to memorize these displays?"

Following this awareness assessment, all participants were informed of the repetition of certain configurations during the task and were told that the generation task would gauge their detection of these repeated configurations. Participants were told that they would see a set of configurations similar to those in the detection task, but that this time the T would be replaced with an L. The instructions for the generation task prompted participants to give the quadrant location of this substitute $\mathrm{L}$ and to respond by using the numeric keypad on the right-hand side of the keyboard. The response layout on the keypad mimicked the spatial layout of the quadrants in the display, with the "7" and "9" keys corresponding to the top left and right quadrants, and the " 1 " and " 3 " keys corresponding to the bottom left and right quadrants. We emphasized that RT was not a priority, and participants were told to concentrate on responding as accurately as possible. A new configuration was shown only after a response to the current display had been made. Configurations were presented continuously without the orienting dot or breaks between blocks of trials. The entire experiment took approximately $50 \mathrm{~min}$ to complete.

\section{Results}

Detection task. One participant was excluded from all analyses because of poor accuracy in identifying the orientation of the target letter during the detection task (75\%). The remaining participants demonstrated high accuracy overall $(M=99 \%, S E=0.14 \%)$, with no difference in accuracy between repeated and nonrepeated configurations $[t(39)=0.39, p=.70]$.

Median RTs were calculated for correct responses. The means of the median RTs for repeated and nonrepeated configurations across the experiment are plotted in Figure 2 and reveal a contextual cuing effect in that RTs were faster to repeated than to nonrepeated displays.

The contextual cuing effect illustrated in Figure 2 was reinforced statistically using a repeated measures ANOVA with configuration (repeated and nonrepeated) and block (1-24) as within-subjects variables. A main effect of block $[F(23,897)=13.36, p<.001]$ indicated that RTs reduced across the blocks. Although the configuration $\times$ block interaction was not significant $[F(23,897)=1.33, p>.1]$, a significant main effect of configuration $[F(1,39)=12.24$, $p=.001]$ confirmed that participants detected targets more rapidly in repeated than in nonrepeated configurations. There was no difference between RTs for repeated and nonrepeated configurations in Block $1[t(39)=0.74, p>.4]$. From these results, one can conclude that substantial and reliable contextual cuing occurred during the detection task.

Generation task. Generation accuracy for each participant was calculated for each configuration type - in each block and overall - then, comparisons were made between configuration conditions in order to evaluate participants' ability to successfully identify the location of the "substitute" L. Chance performance is indicated by no difference in generation accuracy between configuration conditions, because successful generation for nonrepeated configurations is due entirely to chance. Figure 3 plots the mean accuracy scores in each configuration condition for each block of the task. The overall mean for repeated configurations was $30.6 \%$, and that for nonrepeated configurations was $26.1 \%$. A repeated measures ANOVA on generation accuracy using configuration (repeated or nonrepeated) and block (1-4) yielded a significant main effect of configuration $[F(1,39)=8.94, p=.005]$, confirming that higher accuracy ensued for repeated configurations over nonrepeated configurations across the task. Neither the main effect of 


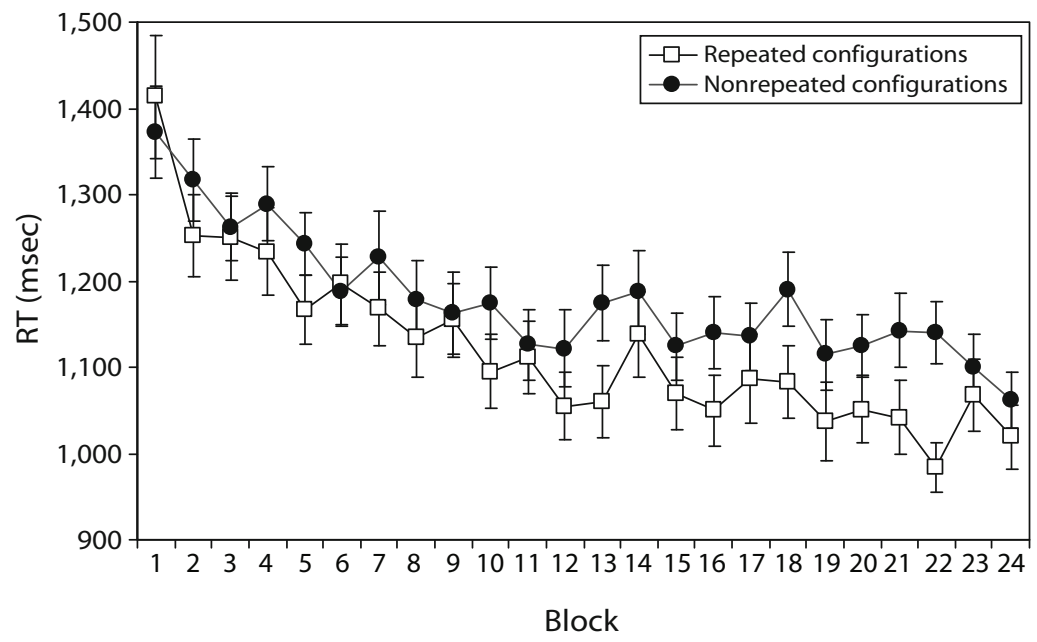

Figure 2. Means of the median reaction time (RT) over 24 blocks of the detection task for repeated and nonrepeated configurations in Experiment 1; error bars show standard errors of the means.

block $[F(3,117)=1.19, p>.3]$ nor the block $\times$ configuration interaction $[F(3,117)=0.65, p>.5]$ approached significance. One-sample $t$ tests comparing generation accuracy for repeated and nonrepeated configurations in all of the blocks of the task with chance performance (25\%) showed that guessing for repeated configurations in each block and overall was significantly above chance [all $t(39) \mathrm{s}>2.0$, all $p s<.05]$, whereas there was no difference between generation for nonrepeated configurations and chance performance [all $t(39) \mathrm{s}<1.58$, all $p \mathrm{~s}>$.10].

Higher accuracy for repeated configurations seems to suggest that participants were aware of the repeating contexts in the detection task. However, the small magnitude of this effect raises the concern that successful generation may have occurred for only 1 or 2 of the configurations learned during detection, whereas contextual cuing itself might occur for many more (perhaps all) of the configura- tions. To address this possibility, we sought to compare the number of repeated configurations showing contextual cuing during detection with the number of consistently generated repeated configurations. Since the configurations were different for each participant, this analysis can only be done at the level of individual participants and cannot be aggregated over configurations. An individual analysis for each of the 12 repeated configurations was conducted for each participant using data from both tasks. The mean RT over the last four blocks of the detection task was computed for each repeated configuration and compared with the participant's mean RT for nonrepeated configurations over these blocks. A repeated configuration was classified as learned if this RT fell below the 99\% confidence interval of the mean nonrepeated RT, indicating that contextual cuing occurred. (We adopted a 99\% interval because the large number [12] of contrasts risks

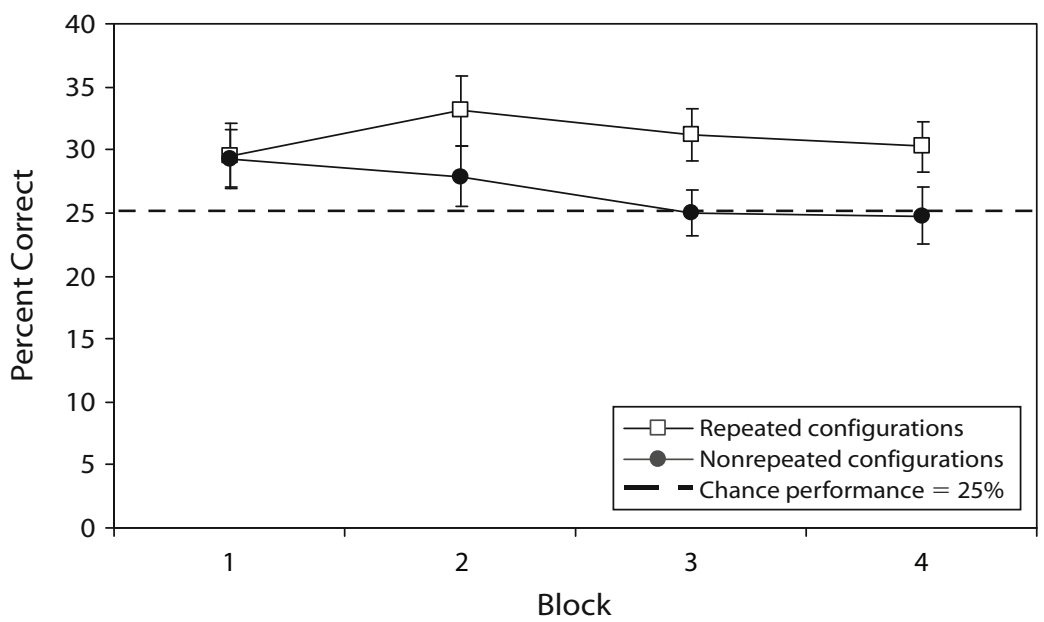

Figure 3. Mean accuracy over the four blocks of the generation task for repeated and nonrepeated configurations in Experiment 1; error bars show standard errors of the means. 
an inflation of the Type I error rate.) This analysis yielded a surprising result - namely, that the mean number of configurations for which contextual cuing occurred was very low $(M=1.55, S D=1.8)$. Thus, on average, a typical participant learned only 1 or 2 configurations $(M d n=1)$. For the generation data, overall accuracy was computed for each repeated configuration. With only four presentations of each pattern, it is somewhat arbitrary to determine when a pattern was "learned" in the explicit test. However, if we take three out of four correct quadrant responses $(75 \%)$ as indicating awareness, then the number of learned patterns $(M=1.55, S D=1.47)$ is very similar to that obtained in the analysis of the contextual cuing effect. If four out of four $(100 \%)$ is the criterion, then the mean number is $0.55(S D=0.99)$.

Figure 4 contains plots of each repeated configuration's RT data against generation performance from high- and low-performing individuals in both tasks. Panel A shows data from the participant with the most configurations showing contextual cuing; panel $\mathrm{B}$, from the participant with the fewest configurations showing contextual cuing. (This is the participant from among 16 with no reliably learned configurations who showed the smallest search advantage for repeated displays in the last four blocks of the task.) Panel $\mathrm{C}$ is for the participant with the highest overall generation performance, and panel $\mathrm{D}$ for the one with the lowest overall generation performance. The figure emphasizes that contextual cuing is not evenly distributed across configurations and is small or negative for many.

With the mean number of implicitly learned patterns being so low, it is hard to argue that more information was acquired during the detection task than was accessed in the generation task. But these results tell us nothing about the correlation between contextual cuing and awareness. We calculated a correlation for each participant between the mean RT of each repeated configuration over the last four blocks of the detection task and percent correct for the same configuration during the generation task. These individual correlations were mostly weak and nonsignificant, and the overall mean correlation ( $z$ score transformed) for all of the participants was also low $(M=0.09)$. However, with a small number of patterns - many of which had generation scores of $50 \%$ - the absence of a significant correlation between these measures is perhaps not particularly diagnostic.
A

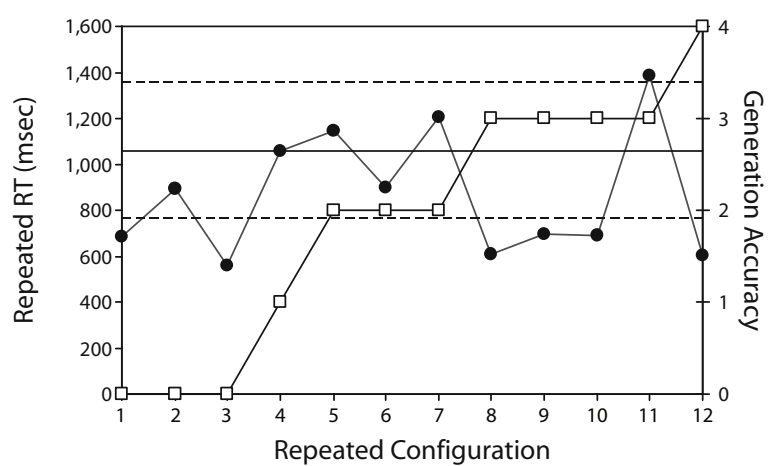

C

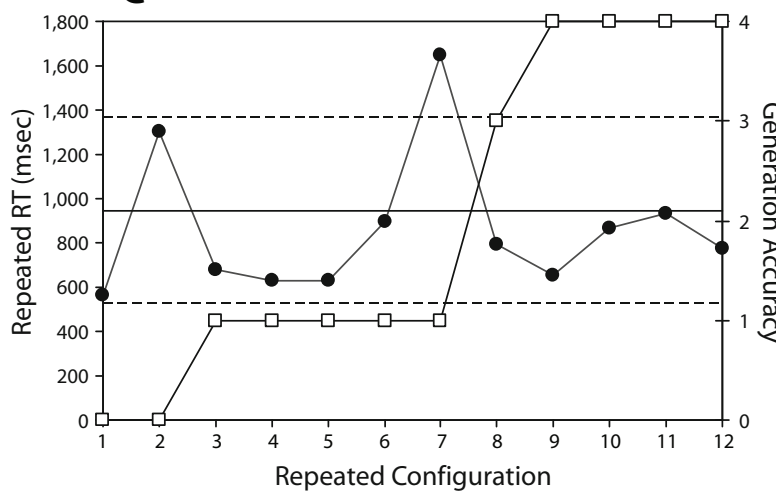

B

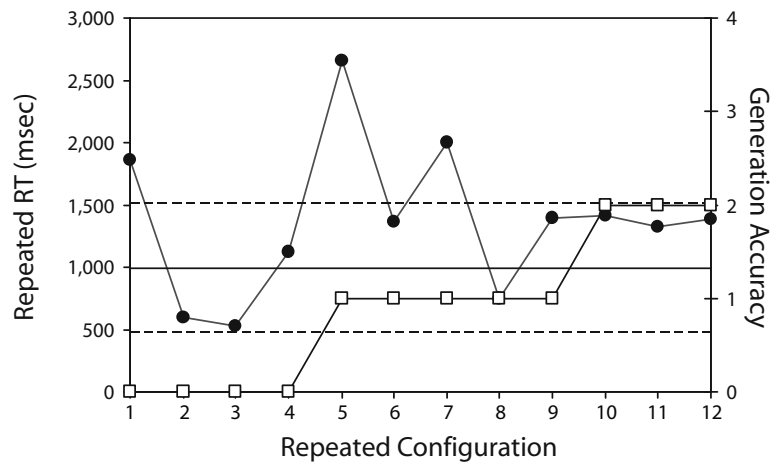

D

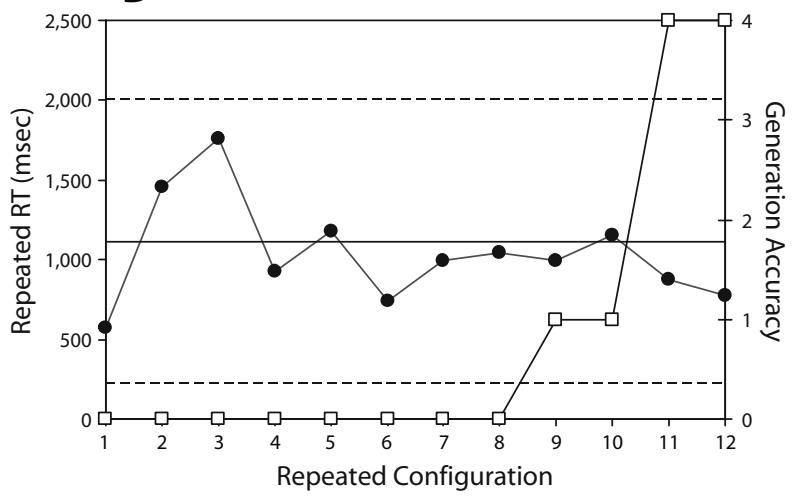

- Repeated configuration RT
- Mean nonrepeated configuration RT
--- Confidence interval of mean (99\%)
$-\square-$ Overall generation accuracy

Figure 4. Graphs plotting individual reaction time (RT) data against generation performance for each repeated configuration (arbitrarily numbered 1-12) from the participant with (A) the most configurations showing contextual cuing, (B) the fewest configurations showing contextual cuing (this is the participant from among 16 with no reliably learned configurations who showed the smallest search advantage for repeated displays in the last four blocks of the task), (C) the highest overall generation performance, and (D) the lowest overall generation performance. 
In order to explore the implicit-explicit correlation further, participants were divided into two groups according to their generation performance. Participants with no overall difference in accuracy between repeated and nonrepeated configurations during generation (i.e., across the 48 repeated patterns in the generation test, they made the same number of or fewer correct target quadrant predictions as they did across the 48 nonrepeated patterns) were assigned to a no-awareness subgroup $(n=17)$, and their data from the last four blocks of the detection task were recalculated. The main effect of configuration $[F(1,16)=$ $3.31, p>.09$ ] was not significant. In contrast, an ANOVA using data from the remaining subgroup of aware participants revealed a main effect of configuration $[F(1,22)=$ $10.51, p<.004]$, indicating that successful generation and detection performance were evident in the aware subgroup, whereas neither contextual cuing nor awareness were present in the unaware subgroup.

Although this result implies that there is a necessary link between learning and awareness, it does not automatically follow that the information explicitly recalled during the generation task accounts entirely for the contextual cuing that was shown during the detection task. For example, it is possible that a contextual cuing effect remains after the removal of configurations for which participants showed explicit awareness (demonstrated by above-chance generation performance). In order to examine this possibility, RT data for a given configuration were removed from an individual's detection data if the participant showed accuracy of greater than chance $(25 \%)$ for that configuration during the generation task. On average, this criterion resulted in the removal of 4 out of 12 configurations from each participant's data set. A reanalysis of the detection data showed that there was still a main effect of configuration $[F(1,39)=7.53, p<.01]$, which suggests that the contextual cuing effect was partly sustained by contextual information for configurations that participants were not subsequently aware of during the generation task. We assess the interpretation of such analyses on the basis of post hoc data selection in the Discussion section.

Despite the evidence of good generation performance, the results do not contradict the original findings from Chun and Jiang (2003). Performance in Block 1, which was equivalent to the entire 24-trial explicit test used by those authors, also showed no difference in generation accuracy between repeated $(29.6 \%)$ and nonrepeated $(28.3 \%)$ configurations $[t(39)=0.40, p>.6]$; however, the fact that higher generation accuracy for repeated configurations was evident with subsequent blocks of trials suggests that previous experiments did not include enough trials to detect the effect. Increasing the number of trials improved the power of the explicit test to show that participants' awareness of the contextual information from the detection task does produce successful memory for repeated configurations during the generation task.

The data do not allow us to determine whether the null result on Block 1 is simply an issue of low power or whether there is a genuine increase in the repeated-nonrepeated effect across blocks (e.g., akin to hypermnesia). The block $\times$ configuration interaction was not significant and, in any case, the change across blocks seems to be due more to a reduction in performance in the nonrepeated condition than to an increase in the repeated one. However, this trend was not supported statistically, since the main effect of block was not significant for generation performance in the nonrepeated condition $[F(3,117)=0.88, p>.4]$.

Reliability analysis. Reliability of the generation task was assessed in a manner similar to the method used in Buchner and Wippich (2000), in which the repeated trials in each block were divided into two subgroups of six trials each (using an odd-even method of assignment) for each individual. Then, the mean generation accuracy was computed for each subgroup of trials and, finally, the means of the subgroups were correlated to evaluate reliability. A high correlation within a set of trials indicates that the task is reliable or, more specifically, that the measure is consistently precise in estimating the participant's awareness of contextual cuing information. Reliability represents the amount of true variance in proportion to observed variance. A measure with low reliability results in data with a higher proportion of error variance, requiring the existing effect to be quite large to reach statistical significance and therefore lowering the statistical power of the measure (Meier \& Perrig, 2000).

Reliability in the first block of generation trials was weak and nonsignificant $(r=.09, p>.5)$, indicating that shorter versions of the generation task used in previous experiments were not reliable. However, when reliability was computed using all 48 repeated generation trials, a strong correlation was found between the means of the subgroups $(r=.46, p=.003)$, confirming that the inclusion of more trials produces a more reliable test and is statistically more powerful than the single-block design used in Chun and Jiang (2003). Reliability was low in the final generation block, as shown by a weak, nonsignificant correlation between measures of accuracy $(r=.19, p>.2)$. This result not only confirmed that measuring generation using a single block of trials is not reliable across the experiment, but also discounted the possibility that a change in participant behavior across blocks was responsible for the awareness effect.

Consistency analysis for the generation task. If participants have explicit knowledge about some configurations, then we should be able to observe consistent responding for such patterns in the generation task. We therefore calculated the likelihood of correctly generating the target location to a given configuration, given that all previous responses to that configuration were correct. If responding is consistent, then this likelihood should increase across repetitions (= blocks), since this would mean that participants gave the same response to a configuration throughout. The probability of a correct response on Block 1 (first presentation) was .30 across all configurations. The probability of a correct response on Block 2-given a correct response on Block 1-was .46. On Block 3, the probability of a correct response-given correct responses on the previous two presentationswas .42. On Block 4, correct responses-conditionalized on correct ones on the previous three presentations-occurred with probability .79. This pattern of increase sug- 
gests that participants adopted consistent response strategies to the patterns they knew. By the fourth presentation, responding was highly accurate (bear in mind that the chance level is .25) for patterns that had evoked correct responses earlier.

Reported awareness results. Awareness for repetitions of configurations was assessed during the interval between the detection and generation tasks. A total of 29 participants $(73 \%)$ reported awareness for the repetition of configurations, with the mean onset of awareness occurring at Block 10 of the search task. Six $(21 \%)$ of these aware individuals reported that they adopted a memorization strategy after the repetition became apparent.

\section{EXPERIMENT 2}

Experiment 1 showed that contextual cuing knowledge is accessible to both implicit and explicit memory, but these results alone do not necessarily provide counterevidence to the claim of dissociation between these memory systems. The design of the experiment does not allow us to make this conclusion, because the explicit test was only presented after the implicit test was concluded; therefore, it shows only that contextual knowledge can be explicitly recalled after it has reached a level at which it can be implicitly recalled. In Experiment 2, we attempted to address this issue by asking whether implicit and explicit accessibility are synchronous when tested concurrently.

The generation task used in Experiment 1 is not the only explicit test that has been employed in previous spatial contextual cuing experiments. Recognition judgments have also been assessed to determine explicit-memory ability (Chun \& Jiang, 1998, 1999; Chun \& Phelps, 1999; Howard et al., 2004; Manns \& Squire, 2001). Chun and Jiang (2003) argued that the generation test was an improvement in several respects (e.g., its response requirements more closely matched those of the detection task). However, because recognition has been used so frequently without being directly compared with generation, we used both tests in the present experiment.

\section{Method}

Participants. Eighty participants (49 women and 31 men) were randomly assigned to the recognition or generation test condition ( $n=40$ per group). All participants were naive to the purpose of the experiment. Roughly half of the participants were not paid for volunteering, whereas the remaining participants ( $n=19$, generation; $n=14$, recognition) received a baseline fee of $£ 4$ and an additional 10 pence for each correct response on explicit test trials.

Design. All participants received an altered version of the detection task from Experiment 1, which was a $2 \times 16$ (configuration type $\times$ block) within-subjects design. The type of explicit task was manipulated between subjects. The generation condition showed only repeated configurations using a four-block repeated measures design and measured accuracy in generating the target location on each trial, whereas the recognition condition was a $2 \times 2$ (configuration $\times$ block) within-subjects design and measured the participant's ability to discriminate repeated configurations from displays not seen previously during the experiment.

Materials and Apparatus. The instructions and procedure described in Experiment 1 were also used for creating and presenting all of the configurations of letters used for this experiment; however, in order to concurrently measure implicit and explicit memory for the learned contextual information, the detection and generationor detection and recognition - tasks were combined into a single procedure. In this new task, generation or recognition trials were presented intermixed with detection trials within an experimental block. In order to accommodate the new concurrent presentation format, the version of the generation task used in Experiment 1 was altered. Generation trials only contained repeated configurations in order to preserve the 4-block design used previously, and also because presenting nonrepeated configurations as generation trials may interfere with the expression of contextual cuing. The detection task was also shortened to just 16 blocks of trials, because a reliable contextual cuing effect can be achieved as early as Block 13 of the detection task, as was shown in Experiment 1.

In the generation group, there were 12 blocks of 28 concurrent trials ( 24 detection and 4 generation). These 12 blocks of concurrent detection and generation trials were also preceded by 3 blocks of just detection trials, since it would not have been useful to measure generation performance before learning had occurred. The 16th and final experimental block was also made up solely of detection trials in order to see whether contextual cuing performance changed in the absence of concurrent explicit assessment.

In 3 blocks of concurrent detection and generation trials, the 12 generation trials cycled through the entire set of repeated configurations. Thus, each individual repeated configuration was shown four times across the 12 concurrent blocks as generation trials. The 4 generation trials in each block contained a repeated configuration with a target location from each quadrant of the screen so that random guessing within each block - and not just across the task overall — would yield chance performance.

The experiment also included a recognition group in which the generation trials were substituted for a recognition task - used previously by Chun and Jiang (1998) to assess explicit memory-which asked participants to discriminate between repeated configurations and novel ones not presented previously during the experiment. This task of detection and recognition also included 12 blocks of 28 concurrently presented detection and recognition trials (24 detection and 4 recognition) and, like the generation condition, these were preceded by 3 blocks and followed by 1 block of pure detection trials. Two repeated and two nonrepeated configurations were shown during the 4 recognition trials so that over the 48 total trials, each repeated configuration was shown twice. The repeated configurations were the same configurations from the detection task, whereas a new set of 24 nonrepeated configurations was created for the recognition portion of the task. These new "recognition nonrepeated configurations" were also generated utilizing the procedures used to create those on detection trials.

Procedure. This new task with concurrent detection and generation-recognition trials began identically to that in Experiment 1 , with instructions to locate the letter $\mathrm{T}$ as quickly as possible within the configuration of letter Ls, followed by 6 practice trials. After receiving three blocks of 24 target-detection trials, participants viewed another set of instructions introducing the explicit test. In order to prevent response delays from changing finger positions (since response requirements changed between trial types), the response keys for the detection task were moved to the numeric keypad, with participants using the left and right arrows on the "4" and "6" keys.

Participants in the generation condition were told that in addition to seeing trials requiring them to quickly locate the $\mathrm{T}$ in the display, the rest of the experiment would also include some other trials (on which they would not be timed) with displays composed entirely of Ls. They were informed that they had seen all of these displays previously during the experiment, but that now an L had been placed where a T would have occurred. Their task was to try to guess which quadrant of the screen contained the "substitute" L. These trials were preceded by a different orientation screen to alert participants of the type of response required on the next trial. Prior to detection trials, participants were shown a blank gray screen with a white dot in the 
center for $1 \mathrm{sec}$ in order to direct their attention to the middle of the screen (as in Experiment 1), whereas the screen shown before generation trials was a black screen with a centered white dot and a red question mark in each quadrant of the display.

In the recognition group, participants were also told that they would be shown additional trials, but that these configurations would appear similar to those seen during detection trials (11 Ls and $1 \mathrm{~T})$. However, on these trials, they were told that they could take their time and decide whether or not they had seen that configuration previously during the experiment. The "7" key on the numeric keypad signaled that they had seen the display or that it was an "old" configuration, whereas the "9" key signaled that they thought the configuration was "new." Participants were alerted to the type of trial to be shown-just as in the generation condition-by the orientation screen.

Auditory feedback was still given on detection trials, but participants did not receive performance feedback on generation or recognition trials. Participants also received a break between blocks of at least $10 \mathrm{sec}$, as in Experiment 1. After all 16 blocks of trials, participants were informed of their performance on the explicit task. The new combined task took about $1 \mathrm{~h}$ to complete in both explicit test conditions.

\section{Results}

Detection task. Participants in both explicit test conditions demonstrated high accuracy overall in responding to the orientation of the $\mathrm{T}$ in the display (generation, $M=$ $97 \%, S E=0.30 \%$; recognition, $M=99 \%, S E=0.14 \%$ ) and showed no difference in response accuracy between repeated and nonrepeated displays [generation, $t(39)=$ $0.29, p>.7$; recognition, $t(39)=0.33, p>.7]$.

Figure 5 shows the results of the detection task for participants in the generation and recognition conditions. Slower detection performance in both configuration conditions was shown in Block 4, which coincides with the introduction of the concurrent presentation of explicit trials in the task. A mixed ANOVA was performed on all of the data, with configuration (repeated vs. nonrepeated) and block (1-16) as within-subjects variables and explicit test (generation vs. recognition) as a between-subjects variable. The results using a shortened detection task replicated performance in Experiment 1, with significant main effects of configuration $[F(1,78)=22.05, p<.001]$ and block $[F(15,1170)=10.58, p<.001]$, and a nonsignificant configuration $\times$ block interaction $[F(15,1170)=$ $1.13, p>.3]$. The significant main effect of configuration is evidence that contextual cuing occurred, since RTs for repeated and nonrepeated configurations were not different in Block 1 for participants in both explicit test conditions [generation, $t(39)=0.09, p>.9$; recognition, $t(39)=1.46, p>.15]$. There were no significant interactions of configuration or block with the explicit test variable (all $F \mathrm{~s}<0.87$, all $p \mathrm{~s}>.6$ ); therefore, one can conclude that equivalent contextual cuing developed in the generation and recognition groups.

The dual-task requirement that participants faced when asked to respond to both detection and generation or recognition trials may have caused contextual cuing in detection trials to be diminished. Greater cuing in Block 16-when only detection trials were presented-would support this idea of the concurrent design of this experiment suppressing the expression of cuing. Accordingly, we compared cuing performance in blocks with and without explicit test trials by taking the difference in RTs between repeated and nonrepeated configurations in Block 15-when detection trials were included with generation or recognition trials-and comparing it with the difference in RTs for configurations when just detection trials were shown in Block 16. There was no difference in the amount of cuing demonstrated by participants between blocks in either explicit test condition [generation, $t(39)=1.32, p>.19$; recognition, $t(39)=0.39, p>.6]$, so the concurrent presentation of the explicit trials alongside detection trials did not appear to interfere with the contextual cuing effect.

Generation task. Generation was solely measured for repeated configurations, so accuracy could only be compared with chance performance. Accuracy averaged across all 12 blocks was significantly higher than chance $(M=$ $29 \%, S E=1.5 \%)[t(39)=2.57, p=.01]$. In order to examine generation performance at different points during the task, accuracy was calculated after each cycle of 12 generation trials presented across 3 blocks of concurrent trials, which was equivalent to performance on 1 block of trials for repeated configurations from the generation task in Experiment 1. A repeated measures ANOVA was performed using generation block (1-4) as a within-subjects variable and revealed no effect for generation block on generation performance $[F(3,117)=0.90, p>.4]$ — which, as illustrated by Figure 6 , shows that above-chance generation performance was sustained throughout the task.

Interestingly, generation performance rose above chance as early as the first cycle of trials $(M=30 \%, S E=2.3 \%)$ $[t(39)=2.33, p<.03]$; however, as shown in Figure 5, this seems to occur before contextual cuing itself was evident. A repeated measures ANOVA was performed on the detection trials corresponding to the presentation of this first cycle of generation trials in Blocks 4-6 and showed a nonsignificant main effect of configuration $[F(1,39)=$ $0.27, p>.6]$, a significant main effect of block $[F(2,78)=$ $9.89, p<.001]$, and a nonsignificant configuration $\times$ block interaction $[F(2,78)=0.43, p>.6]$, confirming that generation ability preceded contextual cuing.

As in Experiment 1, we calculated how many repeated configurations were learned by each participant, focusing on data from the final 4 blocks. This analysis showed that on average, contextual cuing occurred for only 1 of the 12 repeated configurations $(M=0.98, S D=1.53)$. Across all generation trials, participants were able to generate roughly the same number of configurations $(M=0.98$, $S D=1.05,75 \%$ accuracy; $M=0.18, S D=0.35,100 \%$ accuracy), which is convergent with the results obtained in Experiment 1. However, there was a decrease in the number of configurations learned overall in the concurrent task in comparison with Experiment 1, which is most likely related to the shorter task duration used in this experiment.

We next calculated a correlation for each participant between the mean RT of each repeated configuration over the last four blocks of the detection task and percent correct for the same configuration across all generation trials. As before, doing so showed a weak and nonsignificant relationship both on the individual level and overall $(M=0.02)$.

Finally, we divided participants into two groups according to their level of explicit performance and then 

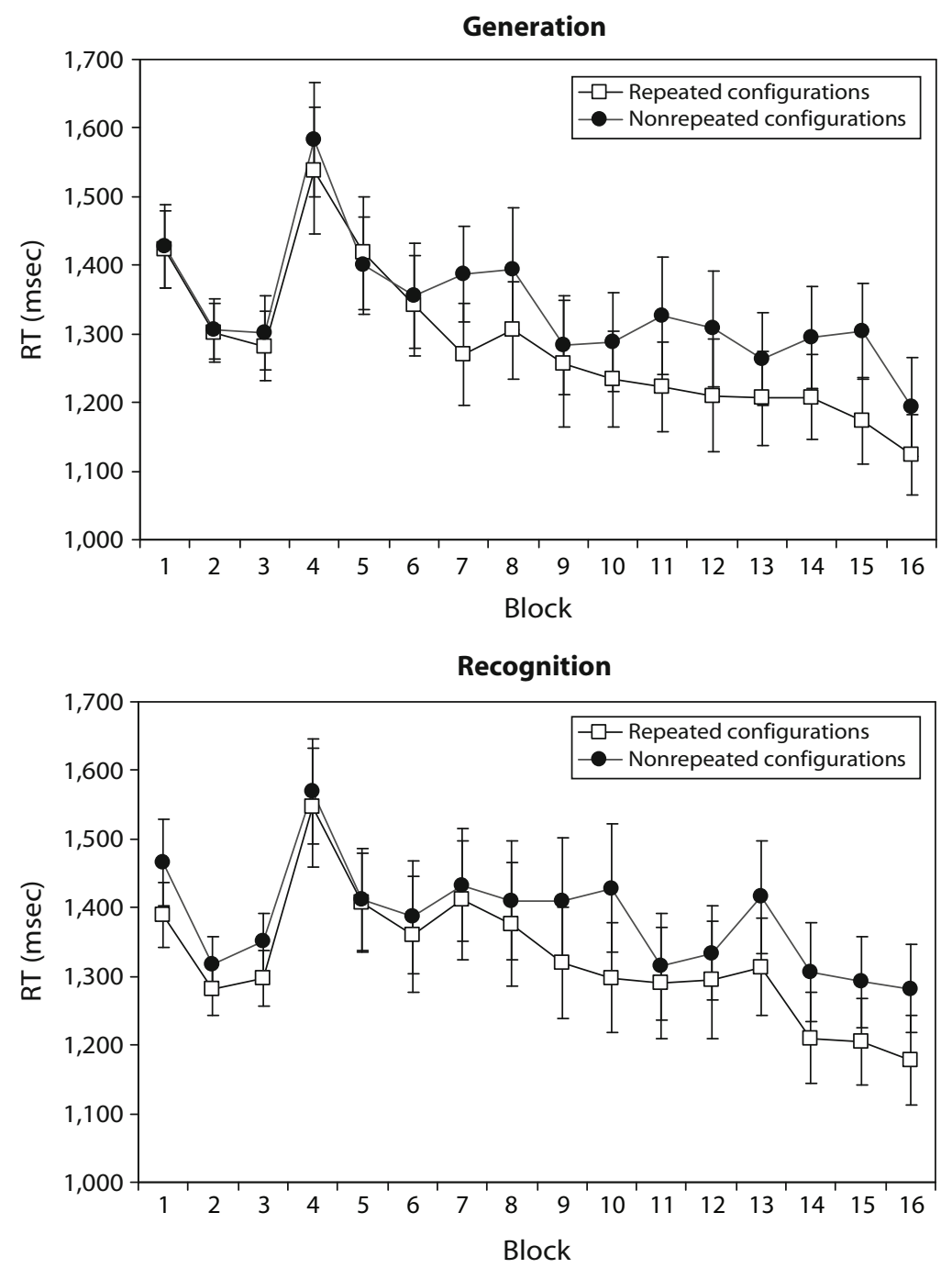

Figure 5. Detection performance over 16 blocks for participants in the generation condition $(n=40)$ and recognition condition $(n=40)$ in Experiment 2. Individual points reflect means of the median reaction time (RT); error bars show standard errors of the means.

reanalyzed their implicit performance. Participants with response accuracy at or below chance level for repeated configurations in the last cycle of generation trials (i.e., across the last 12 generation trials in Blocks $13-15$ of the experiment) were assigned to a no-awareness subgroup $(n=26)$, and their data from the last 4 blocks of the detection task were recalculated. Unlike in Experiment 1, the main effect of configuration $[F(1,25)=13.15, p<.001]$ was highly significant. A comparable analysis using data from the remaining subgroup of aware participants also revealed a (marginally) significant main effect of configuration $[F(1,13)=4.17, p>.06]$. The difference between the groups was not significant. We address the interpretation of this finding in more detail in the Discussion section. (Note that because of the small number of awareness test trials in each block, there is insufficient data to ask whether contextual cuing was reliable just for those configurations showing chance-level explicit knowledge.)
Recognition task. Recognition ability was measured by calculating the hit and false alarm rates for responses to repeated configurations from nonrepeated configurations across recognition trials. A hit is a repeated configuration correctly identified as having been seen before, whereas a false alarm is a nonrepeated display incorrectly identified as having been seen before. Significantly more hits than false alarms indicate that the participant can discriminate repeated from nonrepeated configurations during these explicit trials. The hit rate $(M=.50, S E=.03)$ was significantly higher than the false alarm rate $(M=.41, S E=$ $.03)$ across all 48 trials of the concurrent task $[t(39)=$ $3.68, p<.001]$. In order to see whether performance changed with the presentation of more detection trials, the hit and false alarm rates were also calculated after each cycle (equivalent to six blocks of the concurrent trials) in which all 12 repeated trials had been shown as recognition trials, and the data are shown in Figure 7. After the first 


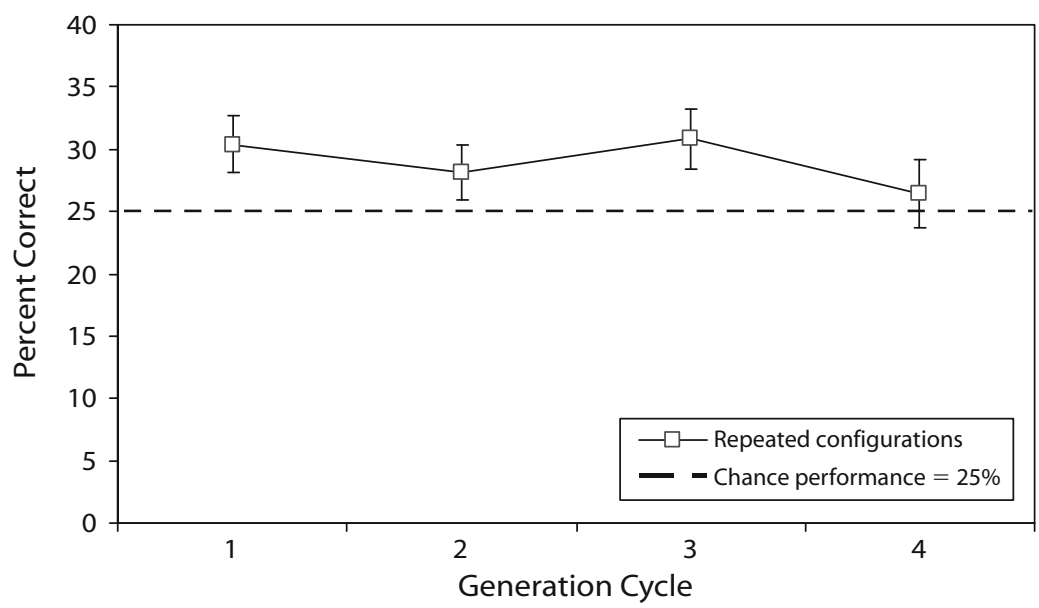

Figure 6. Mean generation accuracy divided up by cycles of generation trials in Experiment 2. One cycle is the point at which 12 generation trials are shown (i.e., all repeated configurations have been displayed); error bars show standard errors of the means.

repetition, the hit rate $(M=.42, S E=.03)$ was not significantly higher than the rate of false alarms $(M=.41, S E=$ $.03)[t(39)=0.40, p>.6]$. Yet, by the second repetition, discrimination ability was evident with a rise in hits $(M=$ $.58, S E=.03)$ versus false alarms $(M=.41, S E=.03)$ $[t(39)=5.13, p<.001]$.

The null result in the first cycle of recognition trials suggests that contextual cuing may have occurred without awareness. In order to investigate this possibility, a repeated measures ANOVA was performed on the corresponding detection trials in Blocks 4-9, and it showed a nonsignificant main effect of configuration $[F(1,39)=$ $2.26, p>.1]$, a significant main effect of block $[F(5,195)=$ $3.31, p<.008$ ] , and a nonsignificant configuration $\times$ block interaction $[F(5,195)=0.42, p>.8]$. Therefore, neither contextual cuing nor recognition ability were present during the first half of the task.
An individual analysis of learning was performed in order to calculate the number of configurations learned in detection and recognition. Participants showed contextual cuing across Blocks 13-16 for approximately one repeated configuration $(M=1.23, S D=1.46)$, which is similar to the amount learned in both the generation task and Experiment 1. For recognition, each pattern was presented only twice across the entire experiment, so our classification took a pattern as having been learned if the correct response was made on both trials. On this basis, the number of configurations learned for recognition was $M=3.5, S D=1.93$, although this result should, of course, be interpreted with caution because of the small number of observations per pattern. As before, the correlation between the mean RT to a repeated configuration during the last 4 blocks of the experiment and the number of correct recognition responses for the same configura-

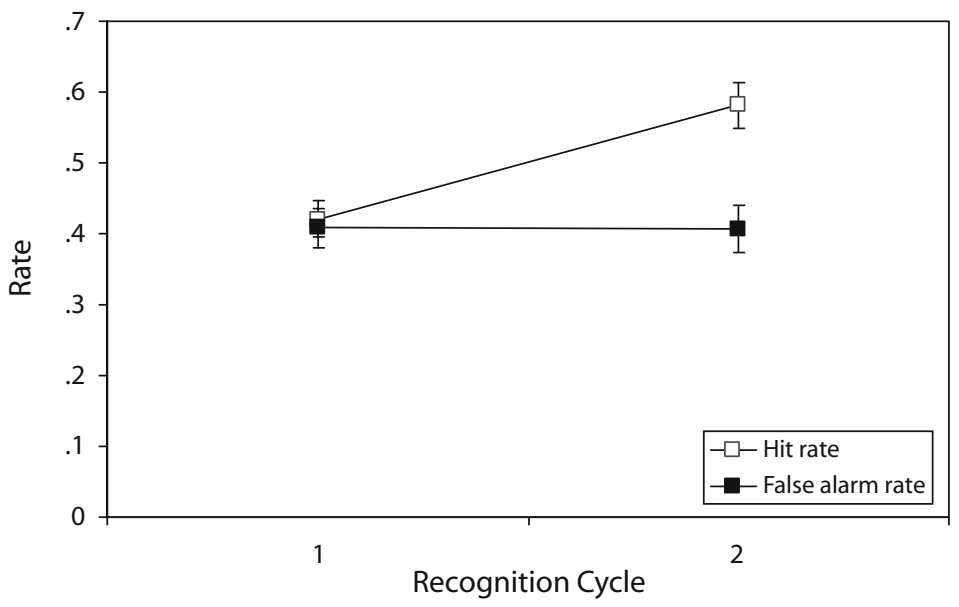

Figure 7. Hits and false alarms segmented by cycles of recognition trials in Experiment 2. One cycle is equivalent to 24 recognition trials comprising all 12 repeated and 12 nonrepeated configurations; error bars show standard errors of the means. 
tion was calculated for each participant. These correlations tended to be nonsignificant, and their $z$-transformed mean was small $(M=0.09)$.

Participants with no difference in recognition accuracy between repeated and nonrepeated configurations in the last cycle of recognition trials (i.e., a negative or zero difference in hit and false alarm rates across the last 12 repeated and 12 nonrepeated patterns shown over Blocks $10-15$ of the experiment) were assigned to a no-awareness subgroup $(n=12)$, and their data from the detection task in Blocks 10-16 were recalculated. The main effect of configuration $[F(1,11)=5.06, p<.05]$ achieved significance. A corresponding analysis on aware participants also revealed a main effect of configuration $[F(1,27)=$ $8.96, p<.006]$, meaning that contextual cuing was present regardless of awareness.

In sum, contextual cuing emerged after approximately 10 blocks of trials. Above-chance generation preceded contextual cuing (being significant across the first three blocks), whereas recognition did not. Thus (1) at a group level, there is no evidence of implicit contextual cuing preceding explicit awareness, and (2) generation seems to be more sensitive than recognition. On the other hand, "unaware" participants did show contextual cuing.

\section{DISCUSSION}

Past research on contextual cuing has found that participants show unconscious facilitation for displays to which they have been exposed repeatedly during a visual search task, which indicates that they have acquired some sort of mental representation of these displays on which they rely to aid their search. However, they do not show evidence of being able to consciously use these representations to support performance during a recognition or generation test (Chun \& Jiang, 1998, 2003; Howard et al., 2004; Manns $\&$ Squire, 2001). This dissociation between unconscious learning and conscious retrieval has led researchers to conclude that the contextual cuing phenomenon illustrates the existence of a purely implicit processing mechanism. The present study was concerned with examining whether this failure to experimentally show conscious access to contextual cuing knowledge is a true effect, or if it is a result of inadequate power and reliability in the methods that the previous studies have used. We implemented a multipleblock design for the explicit test in Experiment 1 and then measured learning and conscious awareness concurrently in Experiment 2. Results from both of our experiments indicate that participants are in fact consciously aware of their contextual cuing knowledge; accordingly, we interpret the null explicit results obtained by past experiments merely as a product of using an insensitive method to measure awareness rather than as a genuine illustration of a dissociation between implicit and explicit systems.

The first experiment directly replicated Experiment 1 of Chun and Jiang (2003), with learning displayed in the 24-block detection task through a marked facilitation in RT for displays repeated throughout the experiment, and accompanied by no ability to correctly generate the location of the transposed target letter for learned displays after 24 trials of generation. In addition, our assertion that it would be difficult to detect a small — but real - explicit effect using this small number of trials was confirmed by the high generation accuracy that emerged in our extended version of the generation task. When we calculated reliability using a single block of trials in the generation task, we found that an individual block of trials was not reliable on its own. Conversely, when this calculation was based on all 4 blocks of generation trials, the measure showed high reliability. Chun and Jiang (2003) found a numerically nonsignificant difference in generation ability between repeated $(27 \%)$ and nonrepeated $(20 \%)$ configurations using a 24-trial task. These authors acknowledged that the null effect may have been due to noisy data; yet they maintained their claim that contextual cuing was a purely implicit process. When our observations about the reliability of generation from Experiment 1 are applied, it is clear that a more plausible explanation for their lack of effect is the low reliability of the measure rather than their participants' actual lack of awareness. Overall, we argue that the shorter version of this task used in past research may not provide a sound measure of participants' true abilities.

A factor that may contribute to the low reliability of the generation measure is the lack of inherent strategic direction given to participants in this task. More specifically, the instructions for the generation task do not guide participants to the best way of attaining the vague and seemingly daunting performance goal of identifying the transformed target letter, using information they do not think they possess. In contrast, the detection task imposes rigid response constraints - for example, to search for the target letter as quickly as possible. Consequently, participants may use a variety of response strategies in the generation task. This variation in task approach decreases the consistency of responses given by participants, which could lead to low reliability of the explicit measure (Buchner \& Wippich, 2000).

An unexpected result of Experiment 1 (confirmed in Experiment 2) was the finding that on average, the contextual cuing effect for a given participant was borne by only one or two configurations. Rather than learning about all or most displays, it seems that a small number of displays evoked fast responses. For a typical participant, many repeated configurations were searched as slowly as were novel ones.

In Experiment 2, we asked whether participants' awareness coincided with their implicit processing by presenting trials from both the explicit and implicit tasks within a single procedure. If contextual cuing knowledge is unable to support both types of processing concurrently, then implicit learning shown in detection performance should precede evidence of conscious access on explicit trials. But participants were able to both respond faster to repeated configurations on detection trials and successfully recognize or accurately generate the target location of altered repeated configurations during the new concurrent task; therefore, it seems that conscious availability of these contextual representations shown from generation or recognition trials coincides with the "unconscious" demonstration of contextual cuing during detection trials. 
Experiment 2 also addressed the difference in sensitivity for contextual cuing information between the two explicit tasks used in contextual cuing experiments. The first experiment using this paradigm by Chun and Jiang (1998) included a recognition task in which discrimination judgments are made on the basis of the participant's sense of familiarity of the displays. Some have argued that in order to conclude that the information supporting contextual cuing is not available to awareness, the test of explicit memory must try to engage the same source driving efficient visual search during the detection task (i.e., cuing of locations by the distractor context). It is not obvious that recognition judgments that are based on display familiarity achieve this. Chun and Jiang (2003) addressed this limitation by introducing the generation task, which requires participants to search the display for the missing target letter and to then respond to its location, thus closely matching the demands of the detection task and in principle making it more sensitive to contextual cuing knowledge. This speculation was confirmed in both experiments. Participants in the generation group showed awareness earlier than did those in the recognition group in Experiment 2, whereas participants in both the Experiment 1 and the Experiment 2 generation conditions showed a roughly equal number of learned individual configurations between the detection and generation tasks.

A somewhat surprising result occurred with the concurrent measurement test design. The generation group showed awareness of contextual knowledge before the contextual cuing effect was evident on detection trials, meaning that awareness seems to have preceded the expression of learning during visual search. Parenthetically, the same outcome has been found in the serial RT task (Perruchet, Bigand, \& Benoit-Gonin, 1997; Shanks \& Johnstone, 1999). Some would argue that the dual-task requirement may have interfered with the expression of contextual cuing knowledge. We doubt that this is a viable explanation, since detection performance did not improve when only detection trials were presented during the last block of the experiment. We believe that the generation effect emerges before contextual cuing, because the explicit test is intrinsically more sensitive than the implicit test. In Experiment 1, a reliable contextual cuing effect was not demonstrated until Block 12 of the detection task, whereas in Experiment 2, a significant overall generation effect was shown after 4 blocks of trials.

Finally, we address the issue of selecting participants or configurations post hoc on the basis of their explicit knowledge and examining performance on the associated implicit test. Numerous studies have analyzed performance in this way by computing the implicit task performance only of participants scoring at or below chance on an explicit measure, or only for trials on which explicit performance was at chance. Here, analyses of contextual cuing in samples of "unaware" participants in both conditions of Experiment 2 (though not of those in Experiment 1) showed reliable learning effects. Likewise, configurations in Experiment 1 that were associated with chance-level generation also showed reliable learning effects. But we argue that these findings still do not constitute clear evidence of im- plicit learning. The reason is that such results are also predicted by single-system models that do not recognize the implicit-explicit distinction (Shanks \& Perruchet, 2002; Shanks, Wilkinson, \& Channon, 2003) and which assume that awareness is a necessary concomitant of learning. Suppose that there is a single knowledge base that controls performance both in an implicit test, such as contextual cuing, and in an explicit test, such as generation. Suppose also, however, that independent sources of noise or error contribute to each performance measure. Under such circumstances, it will inevitably be the case that simulated participants selected after the fact as scoring at or below chance on the explicit measure will score above chance on the implicit measure (Perruchet \& Amorim, 1992), and likewise for configurations selected post hoc on the same basis. Indeed, these models can even predict correlations of 0 between implicit and explicit measures, despite them arising from the same underlying representation (Berry, Henson, \& Shanks, 2006; Kinder \& Shanks, 2003). We believe that for this statistical reason, this form of analysis rarely supports the inferences that are drawn from it.

Note that we are not arguing for a causal role of awareness in learning. Such a conclusion would not be warranted on the basis of our findings. Although there was little evidence of contextual cuing when awareness was absent (except when participants or configurations were selected post hoc-which, we have argued, has its own interpretation), this does not mean that awareness causes learning. An alternative possibility is that learning and awareness are both consequences of a common underlying cause such as a particular type of mental representation (Lovibond \& Shanks, 2002), yielding the slightly weaker conclusion that awareness is a necessary condition for learning.

This research underlines the importance of evaluating the empirical reliability of cognitive measures in these types of experiments. When attempting to demonstrate dissociations between measures of processing, adequate consideration must be given to ensure that both tests have enough power to statistically obtain the effect in question, and caution must be exercised in assuming that the same information is being measured between the tasks. The present study shows that previous dissociations between learning and awareness in contextual cuing emerged because of an insensitive test of awareness, which clearly calls for a revised interpretation of the contextual cuing phenomenon. In conclusion, the results observed in this article suggest that an explanation delineating independent implicit and explicit systems is not necessary to account for contextual cuing.

\section{AUTHOR NOTE}

Correspondence concerning this article should be addressed to A. C. Smyth, Department of Psychology, University College London, Gower Street, London WC1E 6BT, England (e-mail: a.smyth@ucl.ac.uk).

\section{REFERENCES}

Berry, C. J., Henson, R. N. A., \& Shanks, D. R. (2006). On the relationship between repetition priming and recognition memory: Insights from a computational model. Journal of Memory \& Language, 55, 515-533.

Buchner, A., \& WiPPICH, W. (2000). On the reliability of implicit and explicit memory measures. Cognitive Psychology, 40, 227-259. 
Chun, M. M., \& JiAng, Y. H. (1998). Contextual cueing: Implicit learning and memory of visual context guides spatial attention. Cognitive Psychology, 36, 28-71.

Chun, M. M., \& Jiang, Y. H. (1999). Top-down attentional guidance based on implicit learning of visual covariation. Psychological Science, 10, 360-365.

Chun, M. M., \& Jiang, Y. H. (2003). Implicit, long-term spatial contextual memory. Journal of Experimental Psychology: Learning, Memory, \& Cognition, 29, 224-234.

Chun, M. M., \& Phelps, E. A. (1999). Memory deficits for implicit contextual information in amnesic subjects with hippocampal damage. Nature Neuroscience, 2, 844-847.

Dulany, D. E. (1961). Hypotheses and habits in verbal operantconditioning. Journal of Abnormal \& Social Psychology, 63, 251-263.

Dulany, D. E., Carlson, R. A., \& Dewey, G. I. (1984). A case of syntactical learning and judgment: How conscious and how abstract? Journal of Experimental Psychology: General, 113, 541-555.

Howard, J. H., Howard, D. V., Dennis, N. A., Yankovich, H., \& VAIDYA, C. J. (2004). Implicit spatial contextual learning in healthy aging. Neuropsychology, 18, 124-134.

KindER, A., \& SHANKs, D. R. (2003). Neuropsychological dissociations between priming and recognition: A single-system connectionist account. Psychological Review, 110, 728-744.

LoviBOnd, P. F., \& Shanks, D. R. (2002). The role of awareness in Pavlovian conditioning: Empirical evidence and theoretical implications. Journal of Experimental Psychology: Animal Behavior Processes, 28, 3-26.

Maia, T. V., \& McClelland, J. L. (2004). A reexamination of the evidence for the somatic marker hypothesis: What participants really know in the Iowa gambling task. Proceedings of the National Academy of Sciences, 101, 16075-16080.

Manns, J. R., \& SQuire, L. R. (2001). Perceptual learning, awareness, and the hippocampus. Hippocampus, 11, 776-782.

Meier, B., \& Perrig, W. J. (2000). Low reliability of perceptual prim- ing: Consequences for the interpretation of functional dissociations between explicit and implicit memory. Quarterly Journal of Experimental Psychology, 53A, 211-233.

Park, H., Quinlan, J., Thornton, E., \& Reder, L. M. (2004). The effect of midazolam on visual search: Implications for understanding amnesia. Proceedings of the National Academy of Sciences, 101, 17879-17883.

Perruchet, P., \& Amorim, M. A. (1992). Conscious knowledge and changes in performance in sequence learning: Evidence against dissociation. Journal of Experimental Psychology: Learning, Memory, \& Cognition, 18, 785-800.

Perruchet, P., Bigand, E., \& Benoit-Gonin, F. (1997). The emergence of explicit knowledge during the early phase of learning in sequential reaction time tasks. Psychological Research, 60, 4-13.

Shanks, D. R., \& Johnstone, T. (1999). Evaluating the relationship between explicit and implicit knowledge in a sequential reaction time task. Journal of Experimental Psychology: Learning, Memory, \& Cognition, 25, 1435-1451.

Shanks, D. R., \& Perruchet, P. (2002). Dissociation between priming and recognition in the expression of sequential knowledge. Psychonomic Bulletin \& Review, 9, 362-367.

Shanks, D. R., \& ST. John, M. F. (1994). Characteristics of dissociable human learning-systems. Behavioral \& Brain Sciences, 17, 367-395.

Shanks, D. R., Wilkinson, L., \& Channon, S. (2003). Relationship between priming and recognition in deterministic and probabilistic sequence learning. Journal of Experimental Psychology: Learning, Memory, \& Cognition, 29, 248-261.

SQUIRE, L. R. (1992). Memory and the hippocampus: A synthesis from findings with rats, monkeys, and humans. Psychological Review, 99, $195-231$.

Thorndike, E. L. (1931). Human learning. New York: Century.

(Manuscript received November 8, 2006; revision accepted for publication July 24, 2007.) 